\title{
Yield and Failure Criteria for Composite Materials under Static and Dynamic Loading
}

\author{
Isaac M. Daniel \\ Robert McCormick School of Engineering and Applied Science \\ Northwestern University \\ 2137 Tech Drive, Evanston, IL 60208, USA.
}

\begin{abstract}
To facilitate and accelerate the process of introducing, evaluating and adopting of new material systems, it is important to develop/establish comprehensive and effective procedures of characterization, modeling and failure prediction of structural laminates based on the properties of the constituent materials, e. g., fibers, matrix, and the single ply or lamina. A new failure theory, the Northwestern (NUDaniel) theory, has been proposed for predicting lamina yielding and failure under multi-axial states of stress including strain rate effects. It is primarily applicable to matrix-dominated interfiber/interlaminar failures. It is based on micromechanical failure mechanisms but is expressed in terms of easily measured macroscopic lamina stiffness and strength properties. It is presented in the form of a master failure envelope incorporating strain rate effects. The theory was further adapted and extended to the prediction of in situ first ply yielding and failure (FPY and FPF) and progressive failure of multi-directional laminates under static and dynamic loadings. The significance of this theory is that it allows for rapid screening of new composite materials without very extensive testing and offers easily implemented design tools.
\end{abstract}

Keywords: A: Mechanical characterization; B: Dynamic testing; C: Failure criteria; D: Failure envelopes; E: Strain rate effects

Corresponding author. Isaac M. Daniel, e-mail: imdaniel@northwestern.edu, Tel:(847)491-5649, Fax: (847) 491-5227. 


\section{Introduction}

Composite materials in service are exposed to severe loading and environmental conditions which pose new challenges to the designer. In many structural applications composite materials are exposed to high energy, high velocity dynamic loadings producing multi-axial dynamic states of stress. Under these conditions composites exhibit nonlinear and rate-dependent behavior. The process of fabrication, testing and modeling of these composites is costly and time consuming and impedes the introduction of new materials. To facilitate and accelerate the process of introducing and evaluating new composite materials, it is important to develop/establish comprehensive and effective methods and procedures of constitutive characterization and modeling of structural laminates based on the properties of the constituent materials, e. g., fibers, polymers and the basic building block of the composite structure, the single ply or lamina.

Failure of composite materials has been investigated extensively from the physical and phenomenological points of view, on microscopic and macroscopic scales. On the micromechanical scale, failure initiation and failure mechanisms vary widely with type of loading and are intimately related to the mechanical, physical and geometric properties of the constituent phases.

On the macromechanical lamina scale, numerous failure theories have been proposed for analysis of composites and reviewed in the literature [1-12]. The plethora of theories is accompanied by a dearth of suitable and reliable experimental data. A recent development is a new failure theory developed at Northwestern University (NU-Daniel theory) which has been proven very successful in predicting yielding and failure of a composite lamina under multi-axial states of stress and varying strain rates [10-13]. This theory addresses a class of problems where other theories differ the most from each

other. The challenge now is to adapt and extend this new theory to the analysis of progressive failure of multi- 
directional structural laminates under multi-axial static and dynamic loadings and offer easily implemented engineering design tools.

\section{Characterization of Composite Lamina}

Two unidirectional material systems were investigated, AS4/3501-6 and IM7/8552 carbon/epoxy composites. The first one displays quasi-brittle behavior, has been studied more extensively and there is a large body of data available for it. The second system has a higher strength carbon fiber and displays a higher degree of nonlinearity and ductility. Multi-axial experiments were performed by testing unidirectional carbon/epoxy specimens at various loading directions with respect to the principal fiber reinforcement. These experiments produced primarily stress states combining transverse normal and inplane shear stresses.

Experiments were conducted at three strain rates. Quasi-static and intermediate rate tests were conducted in a servohydraulic testing machine at strain rates of $10^{-4} \mathrm{~s}^{-1}$ and $1 \mathrm{~s}^{-1}$, respectively. High strain rate tests were conducted by means of a split Hopkinson (Kolsky) pressure bar at strain rates ranging from 180 to $400 \mathrm{~s}^{-1}$ using prismatic off-axis specimens (Fig. 1). Stress-strain curves were obtained for various off-axis loadings corresponding to different biaxial stress states at various strain rates (Fig.2). The ultimate values provide failure data for various biaxial states of stress.

\section{Strain-Rate-Dependent Failure Criteria}

Most failure theories assume linear elastic behavior and are expressed in terms of macroscopic lamina stresses and strength parameters along the principal material axes. These theories in general can be divided into three categories: (1) Limit or non-interactive theories, such as the maximum stress and maximum strain theories, (2) Fully interactive theories such as the Tsai-Hill and the Tsai-Wu criteria, and (3) Partially interactive or failure mode based theories, such as the Hashin-Rotem, Puck, and NU- 
Daniel theories. The popular fully interactive Tsai-Wu criterion is expressed in the form of a failure polynomial involving all the stress components [1]. The Hashin-Rotem criteria are based on the premise that failure on any plane is only a function of the stress components acting on that plane. Furthermore, separate fiber and interfiber failure modes are considered. The Puck and Shürmann theory is based on the concept of internal friction and a modified Coulomb-Mohr criterion [6]. Sun et al. proposed an empirical modification of the Hashin-Rotem criterion for matrix compressive failure to account for the apparent increase in shear strength due to the transverse compressive stress [2]. Predictions of the various theories, even for a simple unidirectional lamina, can differ a great deal from each other. Failure theories deviate the most from each other for states of stress involving transverse compression and interfiber shear.

The Northwestern (NU-Daniel) interfiber/interlaminar failure theory is based on micromechanical matrix failure mechanisms but is expressed in terms of easily measured macromechanical properties. Three dominant failure mechanisms or modes are identified in a composite element consisting of fibers and interfiber matrix, compression, shear and tension dominated modes [10]. In the compression dominated case, the composite element is loaded primarily in transverse compression with a non-dominant shear component. Failure is assumed to be governed by the maximum (critical) elastic shear strain in the interfiber matrix while the strain along the fiber is constrained to be zero. In the shear dominated case, the composite element is loaded primarily in in-plane shear with a non-dominant compression component. Failure is assumed to be governed by the maximum (critical) elastic tensile strain in the interfiber matrix while constraining the strain component along the fibers. In the tension dominated case, the composite element is loaded primarily in tension with a non-dominant shear component. Failure is assumed to be governed by the maximum (critical) elastic tensile strain in 
the interfiber matrix while constraining the strain component along the fibers. These failure modes are expressed by the following failure criteria:

Compression dominated failure:

$$
\left(\frac{\sigma_{2}}{F_{2 c}}\right)^{2}+\alpha^{2}\left(\frac{\tau_{6}}{F_{2 c}}\right)^{2}=1
$$

Shear dominated failure:

$$
\left(\frac{\tau_{6}}{F_{6}}\right)^{2}+\frac{2}{\alpha} \frac{\sigma_{2}}{F_{6}}=1
$$

Tension dominated failure:

$$
\frac{\sigma_{2}}{F_{2 t}}+\left(\frac{\alpha}{2}\right)^{2}\left(\frac{\tau_{6}}{F_{2 t}}\right)^{2}=1
$$

where $\sigma_{2}$ and $\tau_{6}$ are the transverse (to the fibers) normal stress and in-plane shear stress; $F_{2 \mathrm{t}}, F_{2 \mathrm{c}}$ and $F_{6}$ are the transverse normal tensile and compressive strengths and in-plane shear strength, respectively; $\alpha=$ $E_{2} / G_{12}$ is the ratio of the transverse Young's to the in-plane shear modulus.

Figure 3 shows failure envelopes for a carbon/epoxy composite (AS4/3501-6) under matrix dominated states of stress (transverse compression, transverse tension and in-plane shear). It is shown how the NU-Daniel theory is in very good agreement with experimental results. Similar results were obtained for IM7/8552 carbon/epoxy, which has a much more ductile matrix [12, 13]. The agreement with experimental results is very good. This attests to the robustness of the NU-Daniel theory which is governed by ultimate elastic strains irrespective of the nonlinear elastic and plastic behavior. 
Stress-strain curves to failure of 90-deg and off-axis specimens of the carbon/epoxy composite were obtained as discussed before at three different strain rates (Fig. 2). The basic strength parameters at different strain rates were used and failure envelopes were plotted in Fig. 4 at three strain rates. The

comparison between these failure envelopes predicted by the NU-Daniel theory and experimental results is very satisfactory.

The basic matrix dominated properties of the composite, including the initial transverse and inplane shear moduli, $E_{2}$ and $G_{12}$, the transverse tensile and compressive strengths, $F_{2 \mathrm{t}}$ and $F_{2 \mathrm{c}}$, and the inplane shear strength, $F_{6}$, were obtained from the tests at different strain rates. The strengths, normalized by their quasi-static values, were found to vary linearly with the logarithm of strain rate (Fig. 5). It appears that, for the range of strain rates considered, the variation with strain rate of the matrix dominated strengths can be described as

$$
F(\dot{\varepsilon})=F\left(\dot{\varepsilon}_{o}\right)\left(m \log _{10} \frac{\dot{\varepsilon}}{\dot{\varepsilon}_{o}}+1\right)
$$

where $F=$ strength $\left(\mathrm{F}_{2 \mathrm{t}}, \mathrm{F}_{2 \mathrm{c}}, \mathrm{F}_{6}\right)$

$$
\begin{aligned}
& m=0.057 \\
& \dot{\varepsilon}_{o}=\text { reference strain rate }=\left(\dot{\varepsilon}_{o}=10^{-4} \mathrm{~s}^{-1} \text { for quasi-static loading }\right)
\end{aligned}
$$

In view of the results of Fig. 5 and Eq. (4), the failure criteria of Eqs, (1-3) are recast in a normalized form incorporating the strain rate effects as follows [11]:

Compression dominated failure:

$$
\left(\frac{\sigma_{2}^{*}}{F_{2 c}}\right)^{2}+\alpha^{2}\left(\frac{\tau_{6}^{*}}{F_{2 c}}\right)^{2}=1
$$

Shear dominated failure: 


$$
\left(\frac{\tau_{6}^{*}}{F_{6}}\right)^{2}+\frac{2}{\alpha} \frac{\sigma_{2}^{*}}{F_{6}}=1
$$

Tension dominated failure:

$$
\frac{\sigma_{2}^{*}}{F_{2 t}}+\left(\frac{\alpha}{2}\right)^{2}\left(\frac{\tau_{6}^{*}}{F_{2 t}}\right)^{2}=1
$$

where, $\sigma_{i}^{*}=\sigma_{i}\left(m \log \frac{\dot{\varepsilon}}{\dot{\varepsilon}_{o}}+1\right)^{-1}, \quad \sigma_{i}=\sigma_{2}, \tau_{6}$

and $\quad \alpha=E_{2} / G_{12} \quad$ (independent of strain rate)

Based on the above generalized criteria, the failure envelopes of Fig. 4 collapse into the normalized master envelope shown in Fig. 6.

\section{Strain-Rate-Dependent Yield Criteria}

Before undertaking an analysis of progressive damage in a multi-directional laminate, it is important to understand the initiation of yielding in a lamina, whether isolated or embedded in a laminate. The stress-strain curves of Fig. 2 show a quasi-linear behavior up to a point, yield point, and thereafter become very nonlinear due to plastic deformation. The normal and in-plane shear stress components at yield were fitted to the form of the NU-Daniel failure criteria of Eqs. (1-3) by substituting yield stresses for failure stresses [15]. NU-Daniel yield envelopes and experimental results at three strain rates are shown in Fig. 7. The lamina yield stresses were normalized by their respective quasi-static values and found to vary linearly with the logarithm of strain rate as in the case of failure stresses before $[12,15]$. The linear logarithmic variation is described as follows

$$
F^{y}(\dot{\varepsilon})=F^{y}\left(\dot{\varepsilon}_{o}\right)\left[m \log _{10}\left(\frac{\dot{\varepsilon}}{\dot{\varepsilon}_{o}}\right)+1\right]
$$

where $F^{y}$ is the yield stress $\left(F_{2 c^{y}}{ }^{y}, F_{2 t}{ }^{y}, F_{b}^{y}\right), m$ is 0.092 , and $\dot{\varepsilon}_{o}$ is reference strain rate of $10^{-4} \mathrm{~s}^{-1}$. 
This allowed for transformation of stresses $\sigma_{2}$ and $\tau_{6}$ at any strain rate into equivalent values at the reference strain rate as

$$
\sigma_{i}^{*}\left(\dot{\varepsilon}_{0}\right)=\frac{\sigma_{i}(\dot{\varepsilon})}{f^{y}}
$$

where $\quad f^{y}(\dot{\varepsilon})=\operatorname{mlog}_{10}\left(\frac{\dot{\varepsilon}}{\dot{\varepsilon_{0}}}\right)+1 \quad$ and $\quad \sigma_{i}=\sigma_{2}, \tau_{6}$

The NU-Daniel yield criteria were generalized as follows to account for strain rate dependence:

$$
\begin{array}{ll}
\left(\frac{\sigma_{2}^{*}}{F_{2 c}^{y}}\right)^{2}+\left(\frac{E_{2}}{G_{12}}\right)^{2}\left(\frac{\tau_{6}^{*}}{F_{2 c}^{y}}\right)^{2}=1 \quad \text { Compression Dominated Yield } \\
\left(\frac{\tau_{6}^{*}}{F_{6}^{y}}\right)^{2}+2\left(\frac{G_{12}}{E_{2}}\right)\left(\frac{\sigma_{2}^{*}}{F_{6}^{y}}\right)=1 & \text { Shear Dominated Yield } \\
\left(\frac{\sigma_{2}^{*}}{F_{2 t}^{y}}\right)^{2}+\frac{1}{4}\left(\frac{E_{2}}{G_{12}}\right)^{2}\left(\frac{\tau_{6}^{*}}{F_{2 t}^{y}}\right)^{2}=1 & \text { Tension Dominated Yield }
\end{array}
$$

Based on the above, the yield envelopes of Fig. 7 collapsed into a master yield envelope at the reference strain rate, entirely analogously to the previously described failure envelopes (Fig. 8). Experimental results are in very good agreement with the yield criteria of Eqs. (10-12). The NU-Daniel master yield envelope combined with the previously obtained master failure envelope for this material [12] can serve as lower and upper bounds for design purposes as illustrated in Fig. 9.

\section{Progressive Damage of Composite Laminates}

\section{Yielding of Lamina}

For a given laminate under mechanical and hygrothermal loading, the first stage of damage is the first-ply-yielding (FPY), i. e., the load at which the first ply or group of plies will start yielding or behaving non-linearly. Prediction and characterization of FPY is a challenging task. First-ply-yielding is determined by linear lamination analysis and/or numerical methods, determining the state of stress in 
each individual layer, including fabrication residual stresses. This assumes that a layer or lamina within the laminate exhibits the same properties and behavior as an isolated unidirectional lamina. Residual stresses are calculated using hygrothermal lamination analysis based on the effective temperature difference, which can be estimated as the difference between the service temperature and the glass transition temperature of the matrix resin [8]. The calculated transverse normal and shear stresses in the lamina at yield are described

by the same Northwestern failure criteria, as described before. Lamina yield envelopes were thus obtained for angle-ply laminates of $[ \pm \theta]_{14 \mathrm{~s}}$ layup at various strain rates and plotted in Fig. 10. After noting the linear variation of the normalized yield stresses with the logarithm of strain rate, the yield envelopes collapse into a master yield envelope which is combined with the yield envelope for the isolated lamina of Fig. 8 and shown in Fig, 11.

\section{Failure Initiation and Characteristic Damage State (CDS)}

Following yielding, the lamina is expected to behave in a nonlinear fashion up to failure initiation. In a rigorous analysis a stiffness softening law, $E^{\prime}=\mathrm{f}(E)$, is required to track the behavior of the lamina (and laminae) from yielding up to failure initiation. This is usually in the form of onset of matrix cracking, which is predicted by the selected failure criterion.

Lamina failure within a laminate takes the form of dispersed damage (microcracking) rather than a major localized flaw or crack. This microcracking progresses up to a limiting state or maximum crack density, referred to as the characteristic damage state (CDS). This progressive microcracking is accompanied by lamina (and laminate) stiffness degradation. The stress level at this point is the characteristic damage state stress, $\sigma_{\mathrm{cds}}$, and corresponds to the maximum damage and minimum stiffness

of the layer in the laminate. For the stress-strain curves shown in Fig. 2, the characteristic damage state stress can be determined as the laminate stress when the terminal modulus is reached as shown in Fig. 12. This characteristic damage state stress found experimentally for various angle-ply laminates was 
compared with predictions of the NU failure theory. CDS stress envelopes were thus obtained for various angle-ply laminates at three strain rates as shown in Fig. 13. As before, these envelopes were collapsed into one master envelope (Fig. 14). The agreement with experimental results is satisfactory. 


\section{Summary and Conclusions}

A strain-rate-dependent failure theory (NU-Daniel theory) was described and demonstrated for a composite material. Failure of a composite lamina at various strain rates can be represented by a single master failure envelope. Theoretical predictions are in excellent agreement with experimental results. An entirely analogous theory was described for yield criteria in a composite lamina under multi-axial states of stress at various strain rates. The onset of yielding for various states of stress and strain rates was represented by a single master yield envelope. Again, the predictions of the NU-Daniel yield theory were in very good agreement with experimental results.

The results for an isolated lamina were extended to the behavior of a lamina within a multidirectional laminate. Damage evolution in a laminate was studied using lamination theory and accounting for residual stresses. The state of stress at the onset of yielding and at the damage saturation state (characteristic damage state) was determined for a lamina within a laminate and fitted to the NU theory format. Experimental results for various angle-ply laminates at various strain rates were in good agreement with theoretical predictions.

\section{Acknowledgement}

This work was supported by the Office of Naval Research (ONR). We are grateful to Dr. Y. D. S. Rajapakse of ONR for his encouragement and cooperation.

\section{References}

[1] Tsai, SW, Wu EM. A general theory of strength for anisotropic materials. J. Comp. Materials 1971; 5: 58-80.

[2] Sun CT. Strength analysis of unidirectional composites and laminates. Comprehensive Composite Materials, A. Kelly and C. Zweben (eds), Elsevier Science, Oxford, 2000; 641-666. 
[3] Christensen RM. A survey of and evaluation methodology for fiber composite material failure theories. Mechanics for a New Millennium, H. Aref and J. W. Phillips (eds), Kluwer Academic Publishers, Dordrecht, (2001).

[4] Hinton MJ, Soden PD, Kaddour AS. Failure Criteria in Fibre-Reinforced-Polymer Composites, Elsevier, Oxford, 2004.

[5] Hinton MJ, Kaddour AS, Soden P D. A comparison of the predictive capabilities of current failure theories for composite laminates, judged against experimental evidence. Comp. Sci. Technology 2002; 62: 1725-1798.

[6] Puck A, Shürmann H. Failure analysis of FRP laminates by means of physically based phenomenological models. Comp. Sci. Technology 2002; 62: 1633-1662.

[7] Davila CG, Camanho PP, Rose CA. "Failure criteria for FRP laminates. J. Comp. Materials 2005; 39: 323-345.

[8] Daniel IM, Ishai O. Engineering Mechanics of Composite Materials, Oxford University Press, Second Edition, New York, 2006.

[9] Daniel IM. Failure of composite materials. Strain 2007; 43: 1-9.

[10] Daniel IM, Luo JJ, Schubel PM, Werner BT. Interfiber/interlaminar failure of composites under multi-axial states of stress. Comp. Sci. Technology 2009; 69: 764-771.

[11] Daniel IM, Werner BT, Fenner JS. Strain-rate-dependent failure criteria for composites. Composites Science and Technology 2011; 71: No 3, 357-364.

[12] Schaefer JD, Werner BT, Daniel IM. Strain-rate-dependent failure of a toughened matrix composite. Exper. Mech. 2014; 54: No. 6, 1111-1120.

[13] Daniel IM. Constitutive behavior and failure criteria for composites under static and dynamic loading. Meccanica 2015; 50: No. 2, 429-442.

[14] Werner BT, Schaefer JD, Daniel IM. Deformation and failure of angle-ply composite laminates. Experimental Mechanics of Composite, Hybrid, and Multifunctional Materials, G.P. Tandon et al. (eds.), Springer 2013; 6: No. 19, DOI 10.1007/978-3-319-00873-8_19.

[15] Schaefer JD, Daniel IM. Strain-rate-dependent yield criteria for composite laminates. Fracture, Fatigue, Failure and Damage Evolution, K. Zimmermann (ed.), Springer 2015; 8, Conference Proceedings of the Society for Experimental Mechanics Series, DOI 10.1007/978-3-319-21611-9_26. 


\section{Figure Captions}

Figure 1. High Rate Testing of Composite Specimens in Hopkinson Bar

Figure 2. Stress-Strain Curves for Various States of Stress for AS4/3501-6 Carbon/Epoxy Composite at Three Strain Rates

Figure 3. Failure Envelopes and Experimental Results for AS4/3501-6 Unidirectional Carbon/Epoxy Composite under In-Plane Shear and Transverse Normal Loading [10]

Figure 4. Experimental Results and Failure Envelopes Predicted by the NU-Daniel Theory for AS4/3501-6 Carbon/Epoxy Composite at Three Strain Rates [12]

Figure 5. Variation of Transverse and Shear Strengths with Strain Rate

Figure 6. Master Failure Envelope for AS4/3501-6 Carbon/Epoxy Composite for Strain Rates in the Range of $10^{-4}$ to $450 \mathrm{~s}^{-1}$ [11]

Figure 7. Comparison of NU-Daniel Yield Envelopes with Experimental Results at Three Stain Rates Figure 8. Master Yield Envelope for IM7/8552 Carbon/Epoxy Lamina and Experimental Results

Figure 9. Master NU-Daniel Yield and Failure Envelopes and Experimental Results for IM7/8552 Carbon/Epoxy Composite

Figure 10. Lamina Yield Envelopes and Experimental Results for IM7/8552 Carbon/Epoxy Angle-Ply Laminates at Three Strain Rates [15]

Figure 11. Master Yield Envelope for Lamina and Angle-Ply Laminates in the Range of $10^{-4}$ to $400 \mathrm{~s}^{-1}$ Strain Rates [15]

Figure 12. Stress-Strain Behavior of Lamina within a Laminate Illustrating the Definition of Characteristic Damage State

Figure 13 Envelopes of Characteristic Damage State Stresses for Various Angle-Ply Laminates at Three Strain Rates

Figure 14 Master Envelope for Characteristic Damage State Stresses for Angle-Ply Laminates for Various Strain Rates 


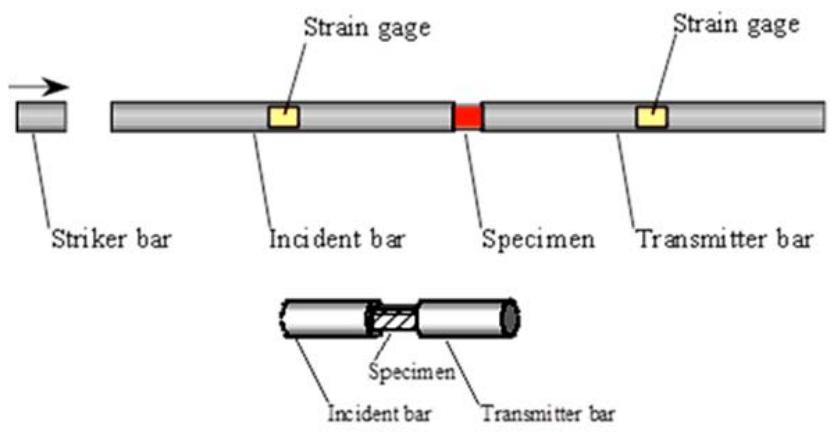

Fig. 1 High Rate Testing of Composite Specimens in Hopkinson Bar
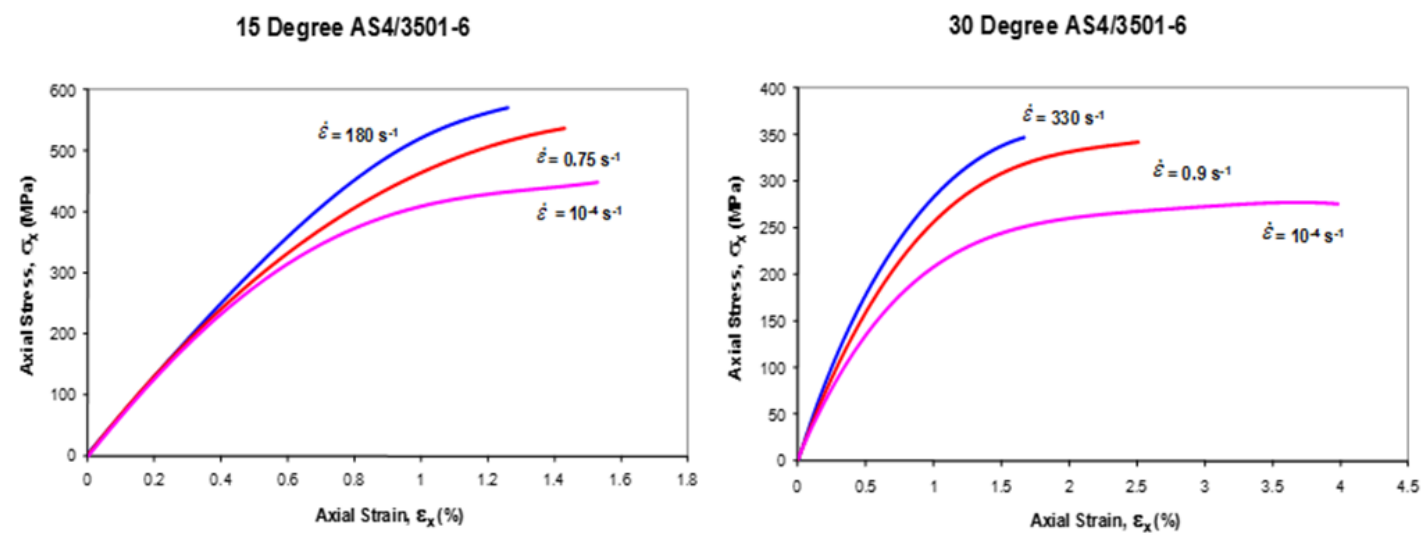

45 Degree AS4/3501-6
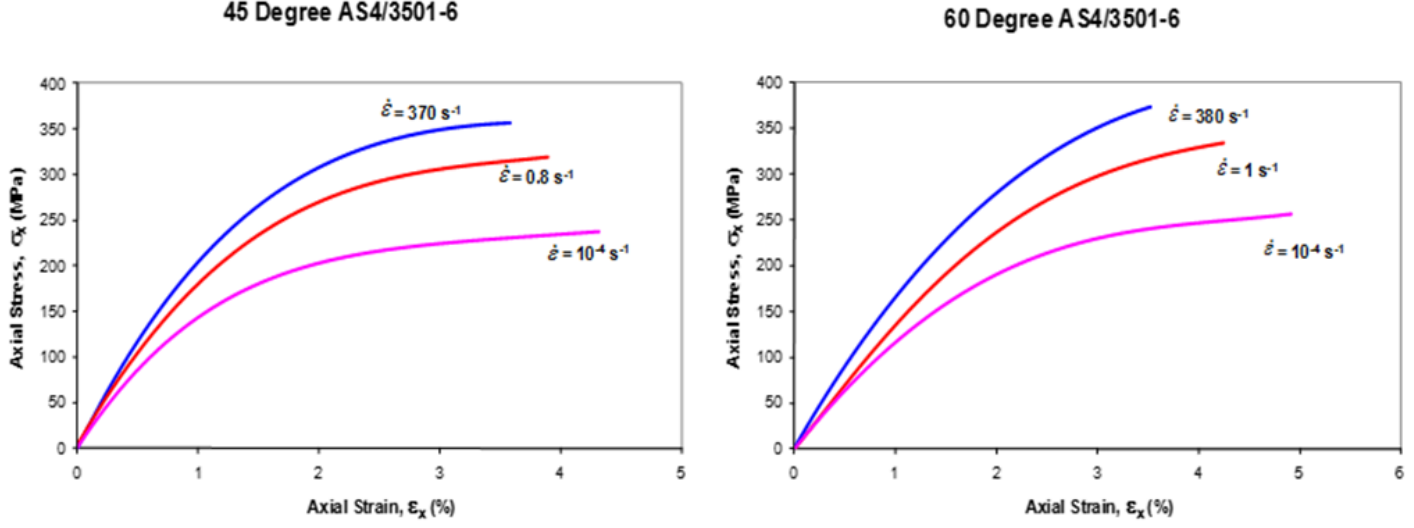

Fig.2 Stress-Strain Curves for Various States of Stress for AS4/3501-6 Carbon/Epoxy Composite at Three Strain Rates 


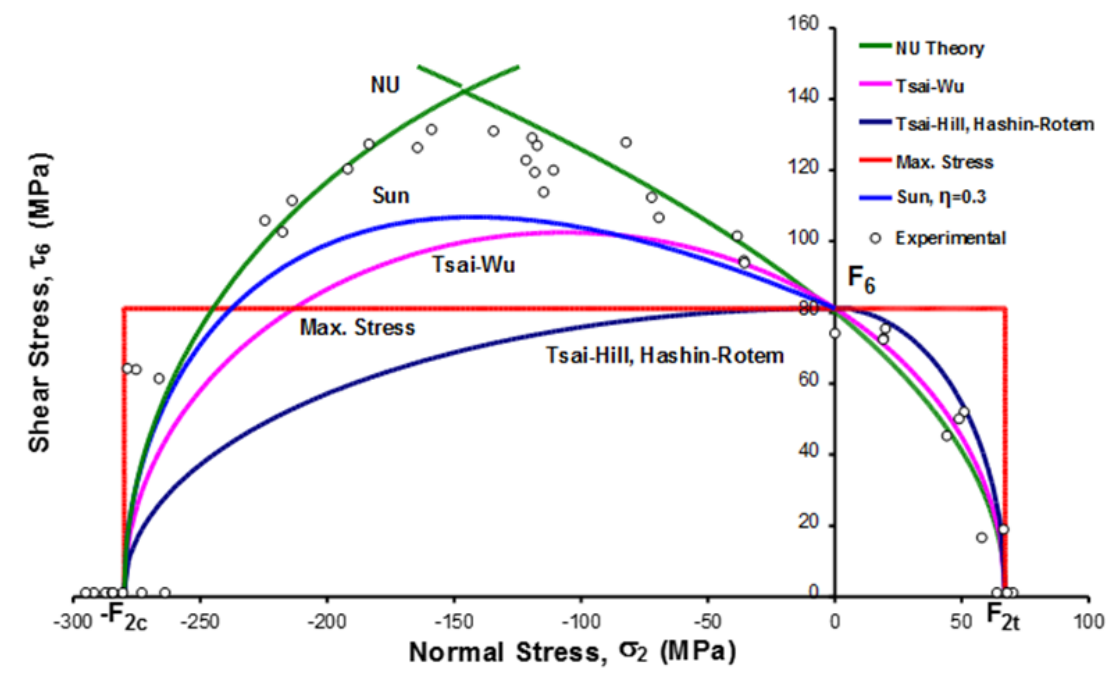

Fig. 3 Failure Envelopes and Experimental Results for AS4/3501-6 Unidirectional Carbon/Epoxy Composite under In-Plane Shear and Transverse Normal Loading [10]

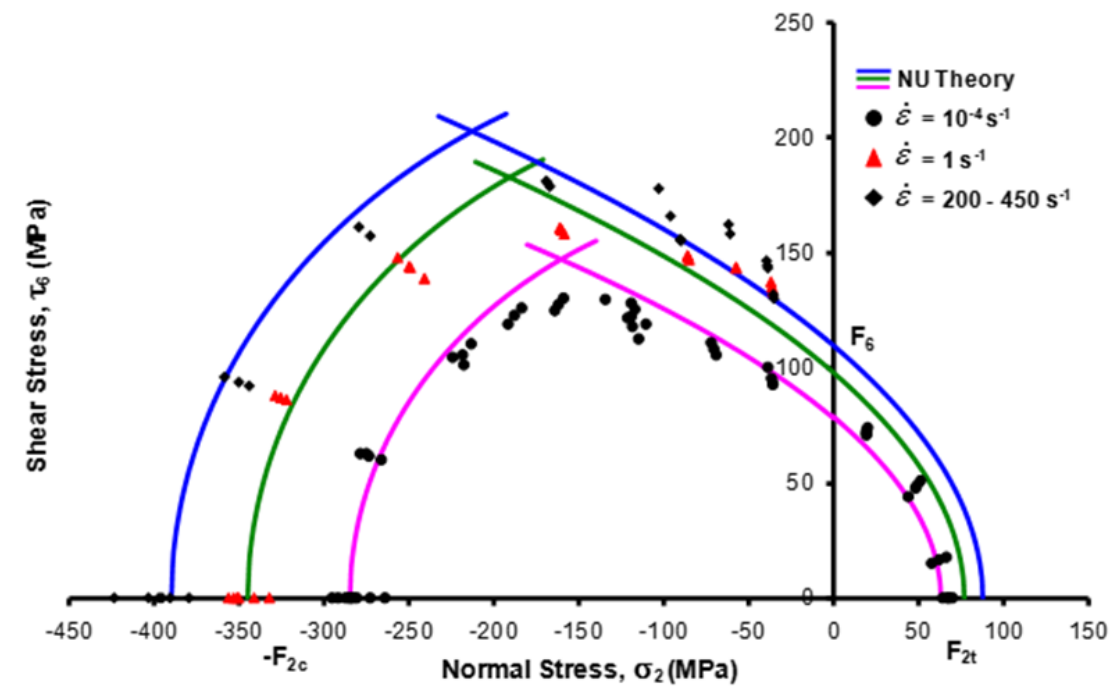

Fig. 4 Experimental Results and Failure Envelopes Predicted by the NU-Daniel Theory for AS4/3501-6 Carbon/Epoxy Composite at Three Strain Rates [11] 


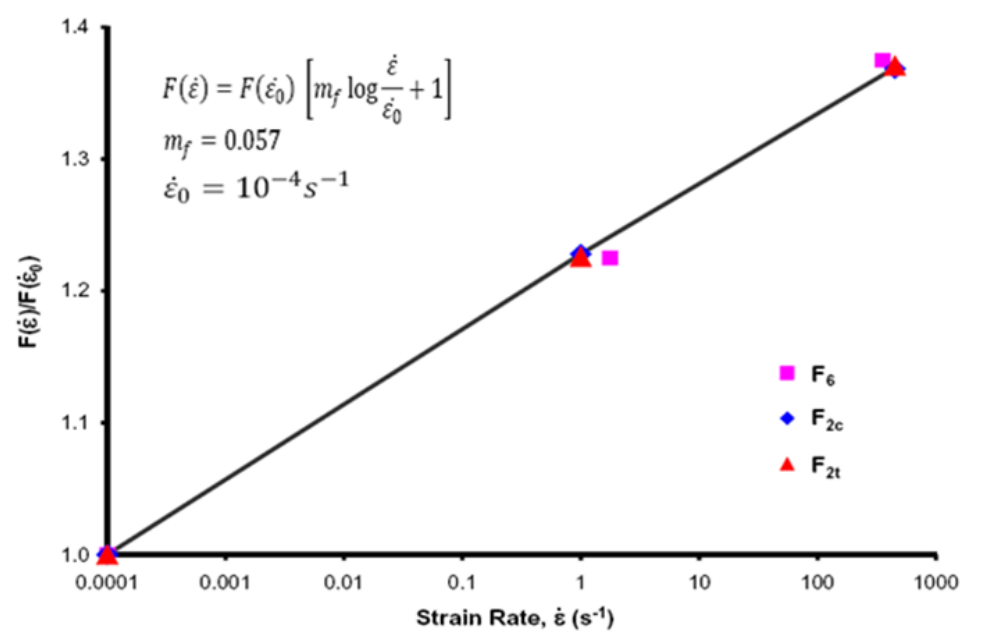

Fig. 5 Variation of Transverse and Shear Strengths with Strain Rate

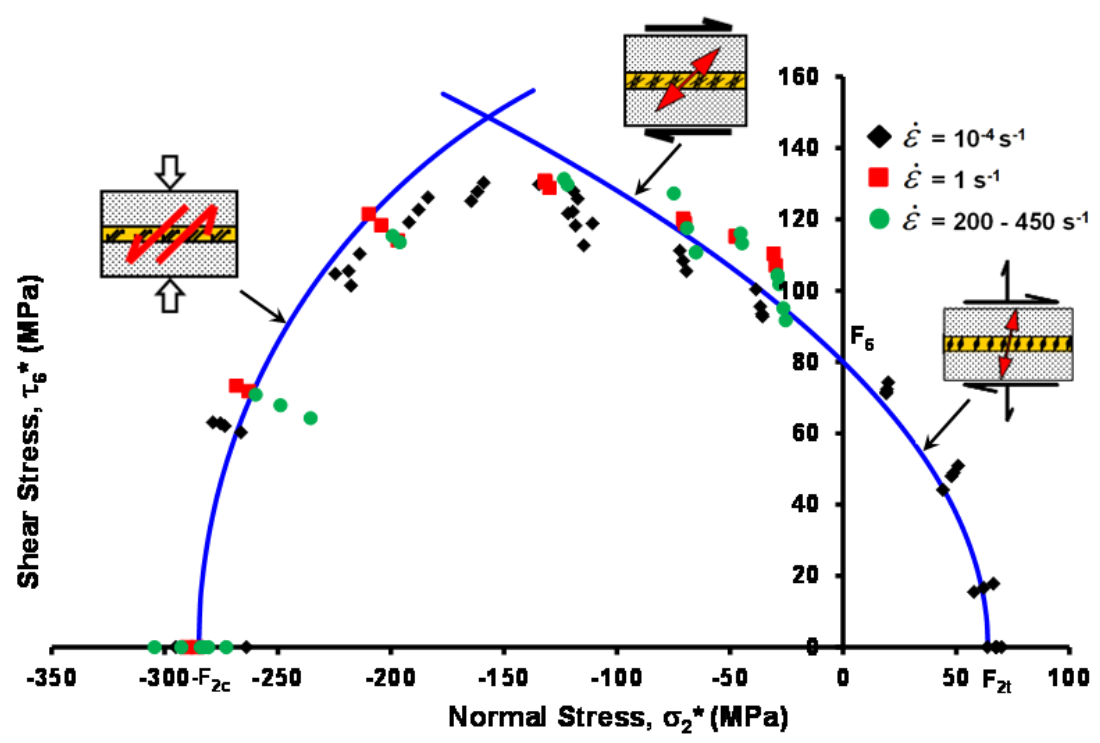

Fig. 6 Master Failure Envelope for AS4/3501-6 Carbon/Epoxy Composite for Strain Rates in the Range of $10^{-4}$ to $450 \mathrm{~s}^{-1}$ [11] 


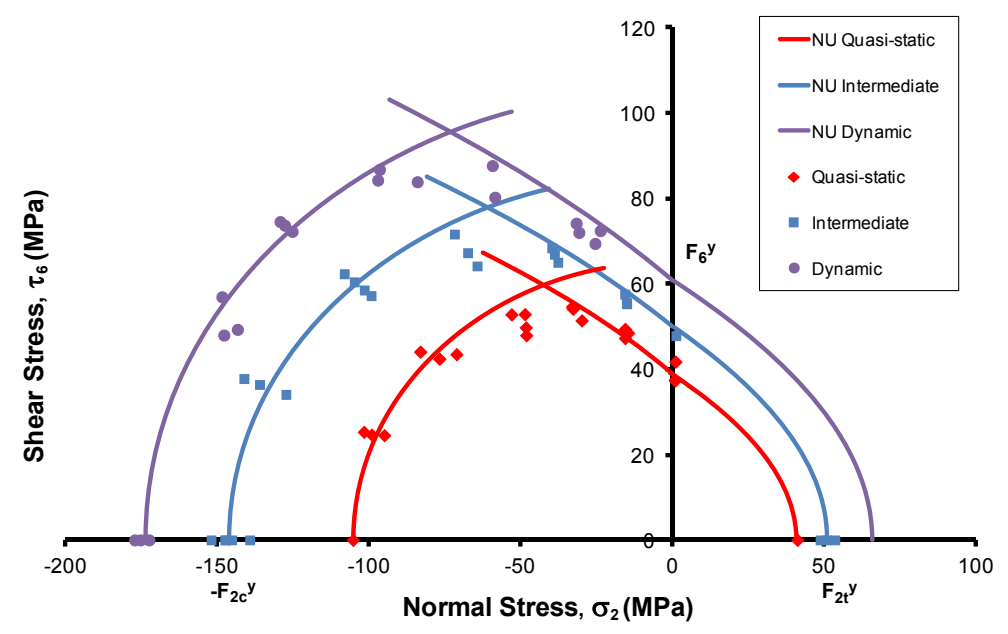

Fig. 7 Comparison of NU-Daniel Yield Envelopes with Experimental Results for IM7/8552 Carbon/Epoxy Composite at Three Stain Rates [15]

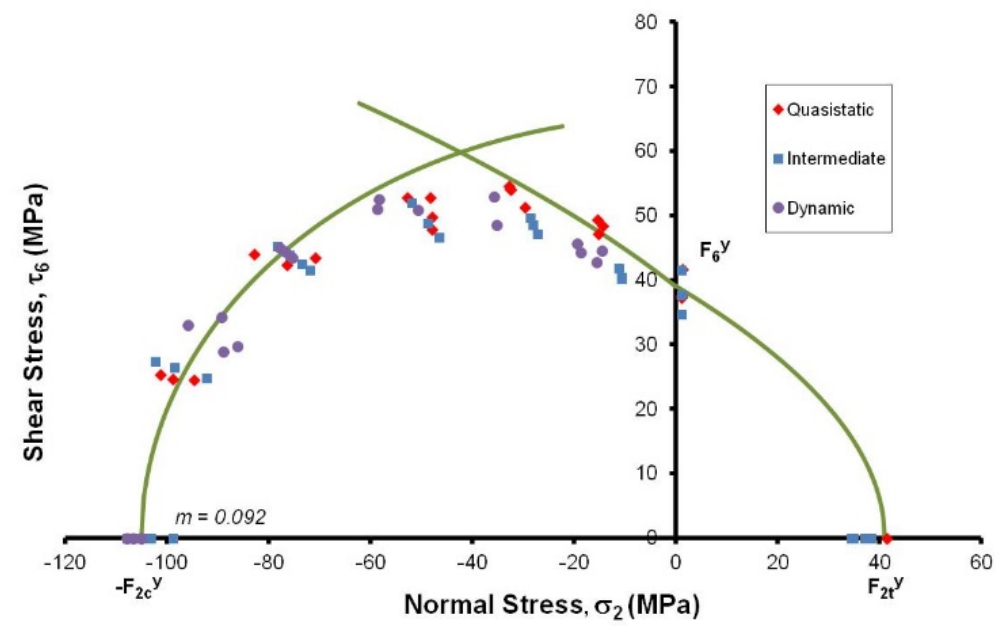

Fig. 8 Master Yield Envelope for IM7/8552 Carbon/Epoxy Lamina and Experimental Results [15] 


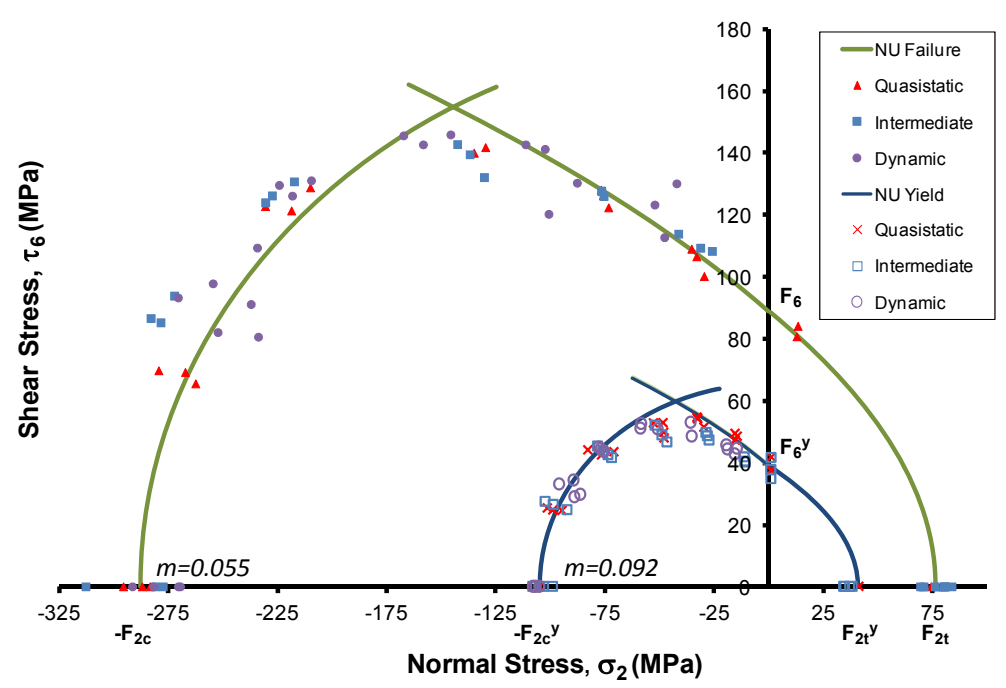

Fig. 9 Master NU-Daniel Yield and Failure Envelopes and Experimental Results for IM7/8552 Carbon/Epoxy Composite

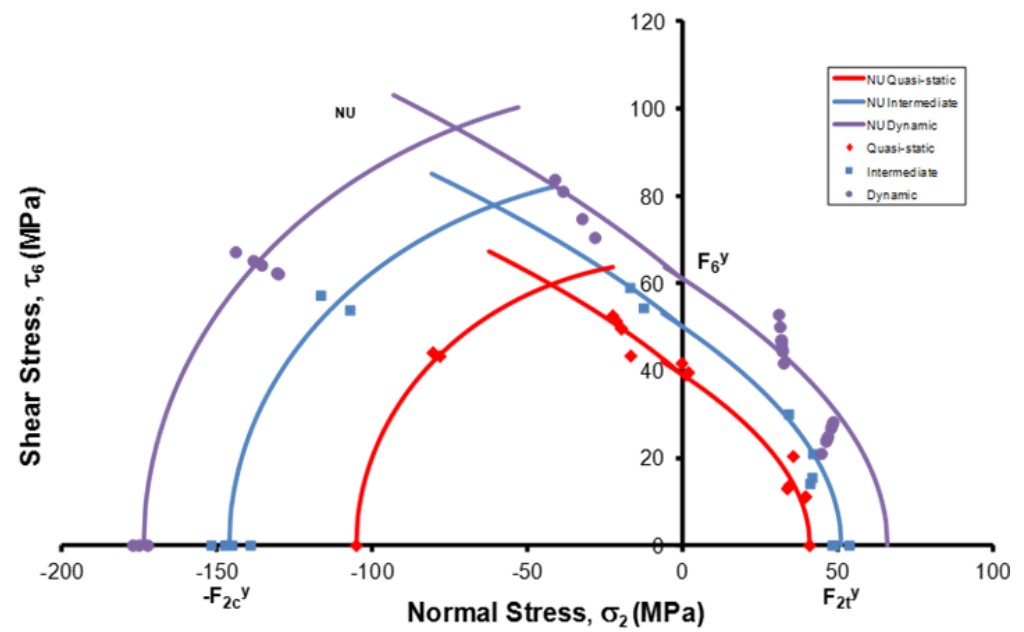

Fig. 10 Lamina Yield Envelopes and Experimental Results for IM7/8552 Carbon/Epoxy Angle-Ply Laminates at Three Strain Rates [15] 


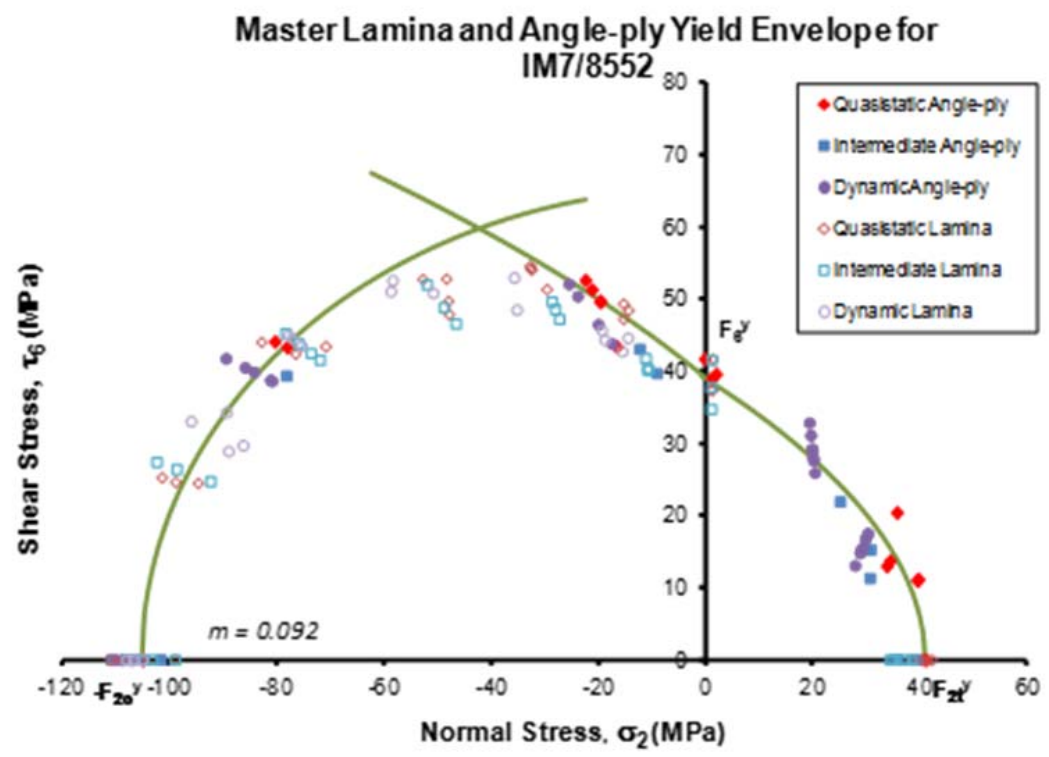

Fig. 11 Master Yield Envelope for lamina and Angle-Ply Laminates in the Range of $10^{-4}$ to $400 \mathrm{~s}^{-1}$ Strain Rates [15]

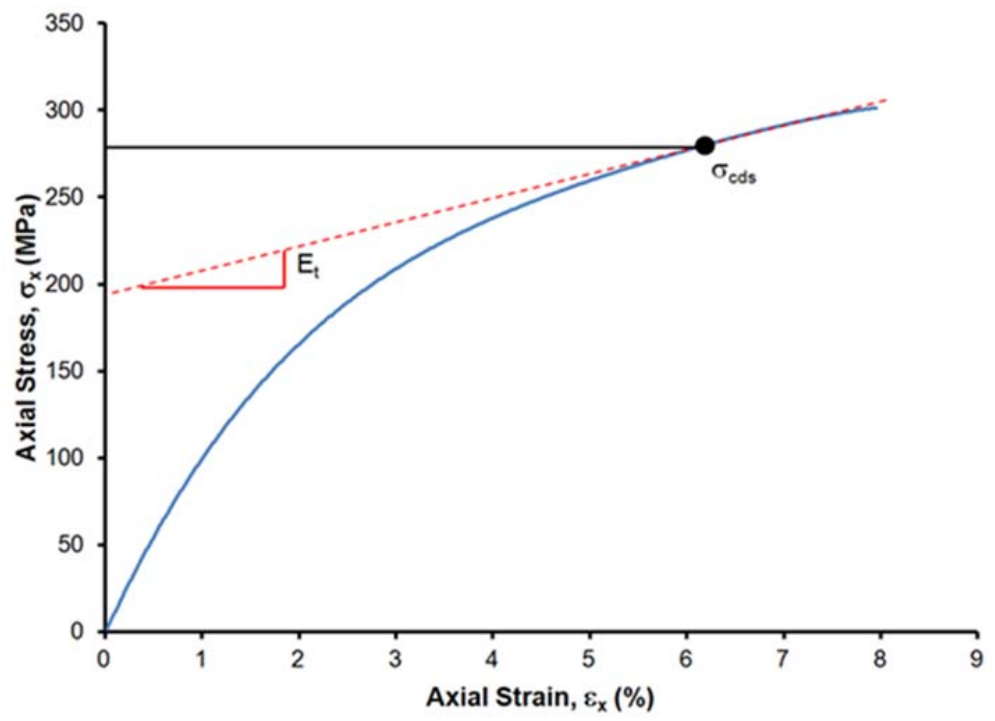

Fig. 12 Stress-Strain Behavior of Lamina within a Laminate Illustrating the Definition of Characteristic Damage State 


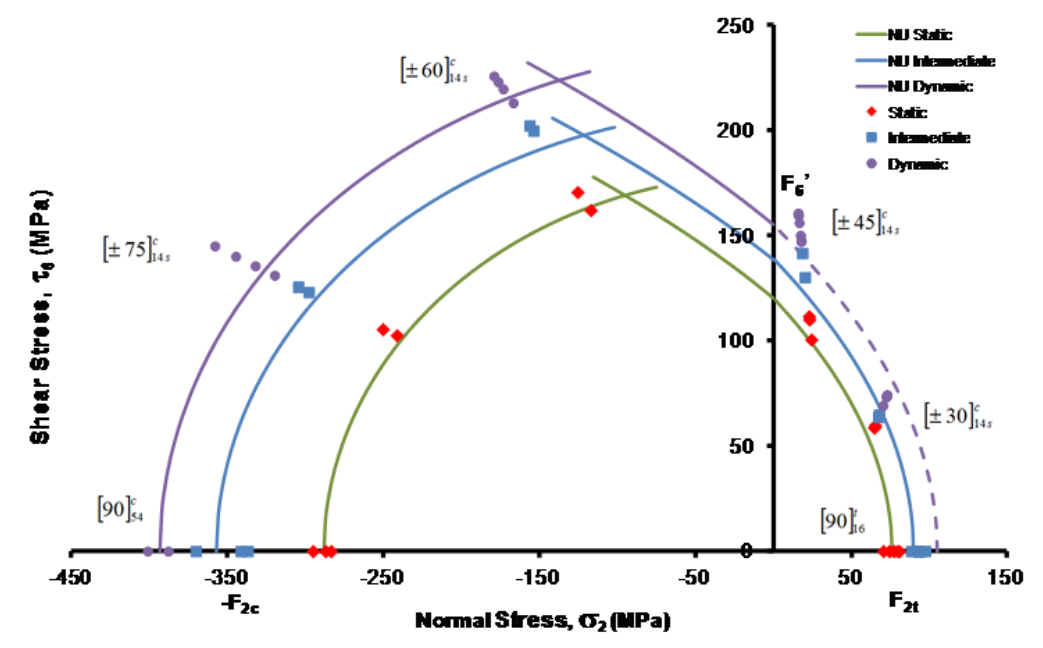

Fig. 13 Envelopes of Characteristic Damage State Stresses for Various Angle-Ply Laminates at Three Strain Rates

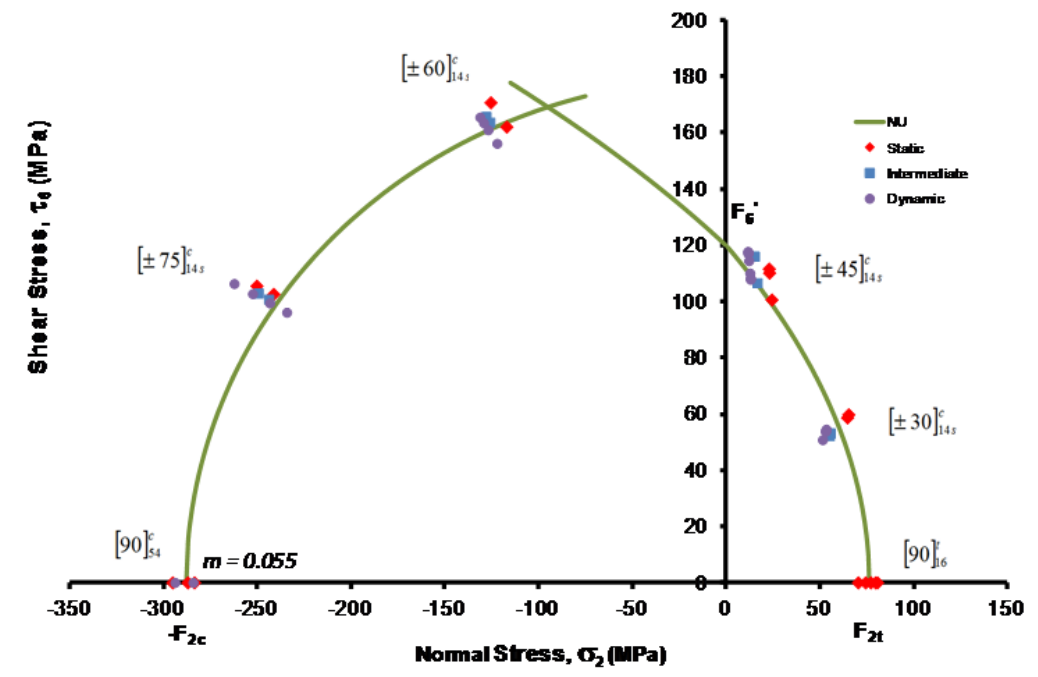

Fig. 14 Master Envelope for Characteristic Damage State Stresses for Angle-Ply Laminates at Various Strain Rates 